\title{
Optical conductivity near finite-wavelength quantum criticality
}

\author{
S. Caprara, M. Grilli, C. Di Castro, and T. Enss \\ SMC-INFM-CNR Rome and Dipartimento di Fisica \\ Università di Roma "La Sapienza", Piazzale Aldo Moro 5, I-00185 Roma, Italy
}

(Dated: November 10, 2018)

\begin{abstract}
We study the optical conductivity $\sigma(\Omega)$ of an electron system near a quantum critical point with finite-wavelength ordering. $\sigma(\Omega)$ vanishes in clean Galilean-invariant systems, unless electrons are coupled to dynamical collective modes, which dissipate the current. This coupling introduces a nonuniversal energy scale. Depending on the parameters of each specific system, a variety of responses arise near criticality: scaling peaks at a temperature- and doping-dependent frequency, peaks at a fixed frequency, or no peaks to be associated with criticality. Therefore the lack of scaling in the far-infrared conductivity in cuprates does not necessarily call for new concepts of quantum criticality.

PACS numbers: 74.25.Gz, 71.45.Lr, 73.20.Mf, 73.43.Nq
\end{abstract}

Quantum criticality has become a central issue in solidstate physics and might account for the non-Fermi-liquid properties of cuprates $1,2,3,4$, heavy fermions ${ }^{5}$ and other strongly correlated electron systems. A relevant goal is to determine the role of critical fluctuations in various anomalous properties. In this context, controversial results on the scaling behavior of optical conductivity $\sigma(\Omega)$ have been found $6,7,8,9,10,11,12$. The issues of scaling violations and the possible need of "concepts beyond the standard model of quantum criticality" $\underline{\underline{6}}$ have been discussed on the basis both of experimental $\underline{\underline{6}}$ and theoretical $\underline{13}$ arguments. In this Rapid Communication we consider the optical conductivity of a system near a quantum critical point (QCP). We show that difficulties in reconciling optical conductivity experiments with the standard quantum criticality framework does not necessarily call for new concepts of quantum criticality, but may naturally arise from the specific nature of this response function: $\sigma(\Omega)$ measures absorption and requires a mechanism for current dissipation (impurities, umklapp processes, dynamical phonons all play this role in real systems). This mechanism, in turn, involves additional energy scales possibly leading to violation of scaling in some parameter range.

Our analysis is carried out for a neutral order parameter with critical collective modes (CM's) at finite wave vectors $\mathbf{q}_{c}$. This is not only pertinent to the cuprates, which are the main object of this work and where a relevant role of spin-ordering $\frac{1}{\underline{\underline{1}}}$ and/or chargeordering ${ }^{2}$ fluctuations has been proposed, but may apply to some heavy fermions, dichalcogenides, and other systems where spatial (usually spin or charge) order occurs at low temperature. The neutral order parameter fluctuations couple in a non-trivial dynamical way with the external electromagnetic field.

We address two issues: (i) to what extent critical CM's, usually characterized by a strong dependence on temperature and other control parameters (like pressure or chemical doping $x$ ), produce specific signatures in $\sigma(\Omega)$ and (ii) how dissipation mechanisms introduce additional scales, which may mask the quantum critical features of optical spectra in some parameter range.

In the first part of this Rapid Communication we con- sider the textbook case of Galilean-invariant electrons, where current dissipation coincides with momentum dissipation. We will later see that the Ward identities enforcing current conservation are in fact more general when implemented for the leading contribution arising from scattering due to CM's at finite $\mathbf{q}_{c}$. In this case our results hold true for electrons in a lattice whenever the umklapp processes for critical scattering are inactive.

- The model - We start from a model effective action at temperature $T$

$$
\begin{aligned}
\mathcal{S} & =T \sum_{k, \sigma} G_{0}^{-1}(k) c_{k, \sigma}^{\dagger} c_{k, \sigma}+T \sum_{q} \chi_{0}^{-1}(q) \phi_{q} \phi_{-q} \\
& +T^{2} \sum_{q} \sum_{k, \sigma, \sigma^{\prime}} \gamma_{\sigma \sigma^{\prime}} c_{k+q, \sigma}^{\dagger} c_{k, \sigma^{\prime}} \phi_{-q},
\end{aligned}
$$

where $k \equiv\left(\mathbf{k}, \epsilon_{\ell}\right), q \equiv\left(\mathbf{q}, \omega_{n}\right), \epsilon_{\ell}\left(\omega_{n}\right)$ are fermionic (bosonic) Matsubara frequencies, $G_{0}=\left(i \epsilon_{\ell}-\xi_{\mathbf{k}}\right)^{-1}$ is the bare quasiparticle $(\mathrm{QP})$ propagator, $\xi_{\mathbf{k}}$ is the dispersion of the QP's created by the $c$ fields, and $\gamma$ is the (theorydependent) vertex coupling the QP's to collective charge or spin excitations, represented by the bosonic fields $\phi$. $\chi_{0}$ is the bare $\mathrm{CM}$ propagator, i.e., the charge or spin susceptibility in the absence of QP polarization dressing. At the bare level the $\phi$ fields mediate an effective electronelectron interaction $V(q)=\gamma^{2} \chi_{0}(q)$. If this interaction is purely static, it cannot lead to electron momentum dissipation in a Galilean-invariant system and, as we show below, $\sigma(\Omega)$ vanishes at any finite frequency, provided it is properly calculated within a conserving perturbative scheme. If instead this bare interaction has its own dynamics arising, e.g., from phonons $\stackrel{2,15}{=}$, electron momentum is dissipated and the optical response is finite. For the sake of concreteness we consider the charge case, where the $\phi$ fields and the $\gamma$ vertex are scalar. To make contact with the case of dichalcogenides and cuprates, we consider a model in two dimensions. We perform a conserving calculation starting from the simplest bubbletype Baym-Kadanoff (BK) functional ${ }^{14}$, which involves only bare QP propagators and the bare interaction $V(q)$ [see Fig. [1(a)]. Once current vertices are inserted, to calculate $\sigma(\Omega)$ by means of the Kubo formula, the bubble diagrams can be resummed by introducing a random- 


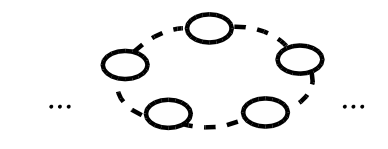

(a)

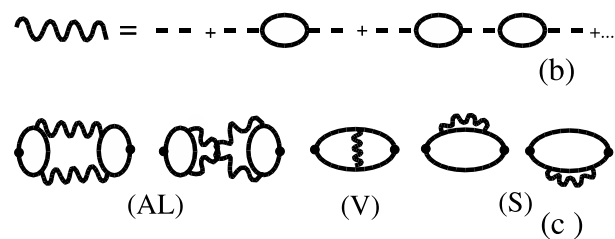

(b)

(AL)

(c)

FIG. 1: (a) Typical diagram of our BK generating functional. The solid and dashed lines represent the QP propagator and the bare interaction $V(q)$, respectively. (b) Bubble resummation to obtain the RPA-dressed CM propagator (wavy line). (c) Diagrams for the current-current response, obtained from current-vertex insertions (solid dots) in the diagrams of the BK functional. AL, V, and S stand for Aslamazov-Larkinlike, vertex, and self-energy diagrams, respectively.

phase-approximation- (RPA) dressed CM propagator [see Fig. [1(b)]. Then, the full set of conserving diagrams reported in Fig. 1(c) is obtained. These diagrams give the $\mathrm{CM}$ corrections to the bare current-current response. In a Galilean-invariant system, $\sigma(\Omega)$ maintains a $\delta$-like Drude term, while the critical CM's may give absorption at finite frequencies.

The RPA effective interaction of Fig. 1(b), $\Gamma=\left(V^{-1}-\right.$ $\Pi)^{-1}$ [here $\Pi(q) \equiv-T \sum_{k} G_{0}(k+q) G_{0}(k)$ is the QP polarization bubble], near the $\mathrm{QCP}$ has the generic form

$$
\Gamma(q)=-\Pi_{\omega}^{-1}\left(m+\varepsilon_{\mathbf{q}}+\left|\omega_{n}\right|+\omega_{n}^{2} / \bar{\Omega}\right)^{-1},
$$

for small frequencies and $\mathbf{q} \approx \pm \mathbf{q}_{c}$. Here, $\Pi_{\omega} \equiv$ $\left[\Pi\left(\mathbf{q}_{c}, \omega_{n}\right)-\Pi\left(\mathbf{q}_{c}, 0\right)\right] /\left.\left|\omega_{n}\right|\right|_{\omega_{n}=0}$, and $\varepsilon_{\mathbf{q}} \approx \nu\left|\mathbf{q} \mp \mathbf{q}_{c}\right|^{2}$. $m, \nu$, and $\bar{\Omega}$ are model-dependent coefficients which result from the second-order expansion of $V^{-1}-\Pi$ around $\mathbf{q}_{c}$ and $\omega_{n}=0$. Except for the $\omega_{n}^{2}$ term, $\Gamma(q)$ has the general hydrodynamic form of a propagator for diffusive CM's (damped by QP's) near a Gaussian QCP. Here $m$, proportional to the square of the inverse correlation length, is the $\mathrm{CM}$ mass measuring the distance from criticality. If the frequency dependence of $\Gamma$ only arises from the QP bubble $\Pi$, i.e., if the bare interaction $V$ is purely static, the optical response is zero [the diagrams of Fig. 1(c) cancel each other], as we show below. On the other hand, if the bare interaction $V$ has its own dynamics, a finite $\sigma(\Omega)$ is obtained. We analyze these two situations, and for concreteness we fix the values of the parameters, adopting as an example the model of Ref. 15. There, a bare interaction $V(q)=V_{0}(\mathbf{q})-\lambda \bar{\omega}^{2}\left(\bar{\omega}^{2}+\omega_{n}^{2}\right)^{-1}$ was considered, arising from both a static short- and long-range Coulomb repulsion $V_{0}$, and from the coupling to a dispersionless phonon of frequency $\bar{\omega}$. For moderate electron-phonon coupling $\lambda \lesssim \varepsilon_{F}$, where $\varepsilon_{F}$ is the typical QP Fermi energy (in cuprates, e.g., $\varepsilon_{F} \approx 0.3 \mathrm{eV}$ ), this interaction can lead to a charge-ordering instability, at a wave vector $\mathbf{q}_{c}$. Within this model we find $m \equiv \Pi_{\omega}^{-1}\left\{\left[\lambda-V_{0}\left(\mathbf{q}_{c}\right)\right]^{-1}+\Pi\left(\mathbf{q}_{c}, 0\right)\right\}$,

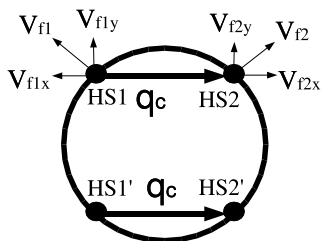

FIG. 2: Sketch of a two-dimensional Fermi surface with four HS's connected by a generic incommensurate critical wave vector $\mathbf{q}_{c}=\left(q_{c}, 0\right)$. Fermi velocities at the HS's and their components are also shown.

and $\bar{\Omega} \equiv \lambda^{-1} \bar{\omega}^{2}\left[\lambda-V_{0}\left(\mathbf{q}_{c}\right)\right]^{2} \Pi_{\omega}$. In Refs. 2 and 15 the instability, signaled by a vanishing $m$, occurred for $\lambda \sim$ $V_{0} \sim\left|\Pi\left(\mathbf{q}_{c}, 0\right)\right|^{-1} \sim \varepsilon_{F}$. Then, we estimate $\nu \sim \varepsilon_{F} / k_{F}^{2}$ $\left(k_{F}\right.$ is the Fermi momentum), and $\bar{\Omega} \sim \bar{\omega}\left(\bar{\omega} / \varepsilon_{F}\right)$.

- Current-current response function - Near criticality, the diagrams of Fig. 1(c), with incoming zero momentum and finite frequency $\Omega_{l}$, are dominated by the poles of the CM propagators. Then, we write the vertex-self-energy (VS) and AslamazovLarkin (AL) contributions to the current-current response function $\chi_{j j}^{\alpha \alpha}$ as $T \sum_{\mathbf{q}, \omega_{n}} \mathcal{V}_{V S}^{\alpha \alpha}\left(\omega_{n}, \Omega_{l}\right) \Gamma\left(\mathbf{q}, \omega_{n}\right)$ and $\frac{1}{2} T \sum_{\mathbf{q}, \omega_{n}}\left[\mathcal{V}_{A L}^{\alpha}\left(\omega_{n}, \Omega_{l}\right)\right]^{2} \Gamma\left(\mathbf{q}, \omega_{n}\right) \Gamma\left(\mathbf{q}, \Omega_{l}+\omega_{n}\right)$, where $\alpha=x, y$ and we have exploited the relation $\Gamma\left(\mathbf{q}, \omega_{n}\right)=$ $\Gamma\left(-\mathbf{q}, \omega_{n}\right)$. The vertices $\mathcal{V}_{V S}^{\alpha \alpha}$ and $\mathcal{V}_{A L}^{\alpha}$ come from the integration on the QP loops. To perform an analytic calculation, we adopt the standard procedure of linearizing the QP dispersion around the points of the Fermi surface connected by $\mathbf{q}_{c}$ [hot spots (HS's)] $\frac{1}{}$. Then, we find (see also Ref. 16)

$$
\begin{aligned}
\mathcal{V}_{V S}^{\alpha \alpha}\left(\omega_{n}, \Omega_{l}\right) & =-\frac{e^{2}}{2} \Pi_{\omega}\left(u^{\alpha}\right)^{2} \Omega_{l}^{-2} \\
& \times\left(\left|\Omega_{l}+\omega_{n}\right|-2\left|\omega_{n}\right|+\left|\Omega_{l}-\omega_{n}\right|\right), \\
\mathcal{V}_{A L}^{\alpha}\left(\omega_{n}, \Omega_{l}\right) & =-i e \Pi_{\omega} u^{\alpha} \Omega_{l}^{-1}\left(\left|\Omega_{l}+\omega_{n}\right|-\left|\omega_{n}\right|\right),
\end{aligned}
$$

where $e$ is the electron charge, $u^{\alpha} \equiv v_{H S 1}^{\alpha}-v_{H S 2}^{\alpha}$, and $v_{H S}^{\alpha}$ is the $\alpha$ component of the Fermi velocity at the HS's (see Fig. 21). The vertices vanish identically in the direction perpendicular to $\mathbf{q}_{c}$, i.e., for $\alpha=y$, since in Fig. 2 we took $\mathbf{q}_{c}$ along the $x$ axis and $v_{H S 1}^{y}=v_{H S 2}^{y}$, i.e., $u^{y} \equiv 0$. Thus $\chi_{j j}^{y y}\left(\Omega_{l}\right) \equiv 0$ for $\Omega_{l} \neq 0$, regardless of the retarded or static character of the bare interaction $V$.

To calculate $\chi_{j j}^{x x}$, we exploit the identity

$$
\Gamma\left(\mathbf{q}, \omega_{n}\right) \Gamma\left(\mathbf{q}, \Omega_{l}+\omega_{n}\right)=\frac{\Pi_{\omega}^{-1}\left[\Gamma\left(\mathbf{q}, \Omega_{l}+\omega_{n}\right)-\Gamma\left(\mathbf{q}, \omega_{n}\right)\right]}{\left|\Omega_{l}+\omega_{n}\right|-\left|\omega_{n}\right|+\frac{\Omega_{l}}{\Omega}\left(\Omega_{l}+2 \omega_{n}\right)},
$$

which is immediately derived from Eq. (11), and allows us to write the AL contribution to $\chi_{j j}^{x x}$ as

$$
-T \sum_{\mathbf{q}, \omega_{n}} \frac{\Pi_{\omega}^{-1}\left[\mathcal{V}_{A L}^{x}\left(\omega_{n}, \Omega_{l}\right)\right]^{2}}{\left|\Omega_{l}+\omega_{n}\right|-\left|\omega_{n}\right|+\frac{\Omega_{l}}{\Omega}\left(\Omega_{l}+2 \omega_{n}\right)} \Gamma\left(\mathbf{q}, \omega_{n}\right),
$$

where a single effective interaction appears, at the expense of a more complicated prefactor.

It is instructive to consider first the case $\bar{\Omega} \rightarrow \infty$, (i.e., $\bar{\omega} \rightarrow \infty$ ). In this case the QP's are only coupled 
among themselves and with a static phonon field, and the frequency dependence in Eq. (1) only comes from the QP bubble. As a consequence, $\sigma(\Omega)$ must vanish for any finite frequency, while only the $\delta$-like Drude term remains. For $\bar{\Omega} \rightarrow \infty$ and by means of Eq. (3), Eq. (4) can be cast in the form of a Ward identity $e u^{\alpha}\left[\Gamma\left(\mathbf{q}, \Omega_{l}+\right.\right.$ $\left.\left.\omega_{n}\right)-\Gamma\left(\mathbf{q}, \omega_{n}\right)\right]=i \Omega_{l} \mathcal{V}_{A L}^{\alpha}\left(\omega_{n}, \Omega_{l}\right) \Gamma\left(\mathbf{q}, \Omega_{l}+\omega_{n}\right) \Gamma\left(\mathbf{q}, \omega_{n}\right)$, which plays the role of the ordinary Ward identity exploited, e.g., in Ref. 17, to prove the cancellation of the paraconductivity, in the case when the electron momentum commutes with the Hamiltonian. In our case, by means of Eqs. (21) and (31) we can eliminate the VS vertex in favor of the AL vertex, $\mathcal{V}_{V S}^{x x}\left(\omega_{n}, \Omega_{l}\right)=$ $-\frac{i e}{2} u^{x} \Omega_{l}^{-1}\left[\mathcal{V}_{A L}^{x}\left(\omega_{n}, \Omega_{l}\right)+\mathcal{V}_{A L}^{x}\left(-\omega_{n}, \Omega_{l}\right)\right]$. Then, $\chi_{j j}^{x x}=$ $\frac{i e}{2} u^{x} \Omega_{l}^{-1} T \sum_{\omega_{n}, \mathbf{q}}\left[\mathcal{V}_{A L}^{x}\left(\omega_{n}, \Omega_{l}\right)-\mathcal{V}_{A L}^{x}\left(-\omega_{n}, \Omega_{l}\right)\right] \Gamma\left(\mathbf{q}, \omega_{n}\right)$ vanishes identically, since the quantity between square brackets is an odd function of $\omega_{n}$.

- Momentum dissipation: finite $\bar{\Omega}$ - To obtain an optical response it is therefore necessary to introduce a dissipation mechanism. In this Rapid Communication we implement this dissipation via the $\omega_{n}^{2}$ term in Eq. (1), which in the example we are considering arises from the phonon dynamics. In this case, after analytic continuation of the external Matsubara frequency, we find

$$
\begin{aligned}
\chi_{j j}^{x x}(\Omega) & =\frac{A}{(i \Omega-\bar{\Omega}) \Omega} P \int_{-\infty}^{+\infty} \frac{d z}{i \pi} \ln \left[\frac{z^{2}-\bar{\Omega}(\Lambda-i z)}{z^{2}-\bar{\Omega}(m-i z)}\right] \\
& \times\left[\frac{\bar{\Omega} \Omega+i(2 z+\Omega)(z-\Omega)}{\bar{\Omega}-i(2 z+\Omega)} \operatorname{coth}\left(\frac{z}{2 T}\right)\right. \\
& \left.-\frac{i(2 z-\Omega)(z-\Omega)}{\bar{\Omega}-i(2 z-\Omega)} \operatorname{coth}\left(\frac{z-\Omega}{2 T}\right)\right]
\end{aligned}
$$

where $A=e^{2}\left(v_{H S}^{x}\right)^{2} / \nu d$ is the dimensional prefactor, $d$ is the interlayer distance which translates the twodimensional response into the in-plane response of a layered system, and $\Lambda$ is an ultraviolet cutoff. Extracting the conductance quantum $e^{2} / h$ and the factor $1 / d$, we are left with a dimensional factor which we estimate as $h\left(v_{H S}^{x}\right)^{2} / \nu \sim \varepsilon_{F} / h$.

One can check that $\operatorname{Im} \chi_{j j}^{x x}(\Omega)$ linearly vanishes with $\Omega$, giving a finite $\sigma(\Omega)=\operatorname{Im} \chi_{j j}^{x x}(\Omega) / \Omega$, for $\Omega \rightarrow 0$. However, in our calculation without disorder, a $\delta$-like Drude term $\left[D_{0}-\pi \operatorname{Re} \chi_{j j}^{x x}(0)\right] \delta(\Omega)$ is still present, where $D_{0}$ is the QP Drude weight in the absence of the CM contribution. Kramers-Kronig relations connecting $\operatorname{Re} \chi_{j j}^{x x}$ and $\operatorname{Im} \chi_{j j}^{x x}$ guarantee spectral weight conservation within our conserving approach: the finite-frequency weight associated with $\sigma(\Omega)$ is exactly subtracted from $D_{0}$ by $\pi \operatorname{Re} \chi_{j j}^{x x}(0)$, i.e., $\int d \Omega\left\{\left[D_{0}-\pi \operatorname{Re} \chi_{j j}^{x x}(0)\right] \delta(\Omega)+\sigma(\Omega)\right\}=D_{0}$.

In Eq. (5), the $\bar{\Omega}$ dependence is crucial and introduces a non-critical energy scale in the absorption, which may strongly alter the dependence of the optical spectra on the CM mass. The scale $\bar{\Omega}$ (which we estimated for the cuprates as a rather low energy scale $\sim \bar{\omega}^{2} / \varepsilon_{F}$ ) determines, at low $T$, the frequency below which the response tends to be vanishingly small, as it is for $\bar{\Omega}=\infty$. On the other hand, for $\Omega \gtrsim \bar{\Omega}$ a finite absorption is found. We

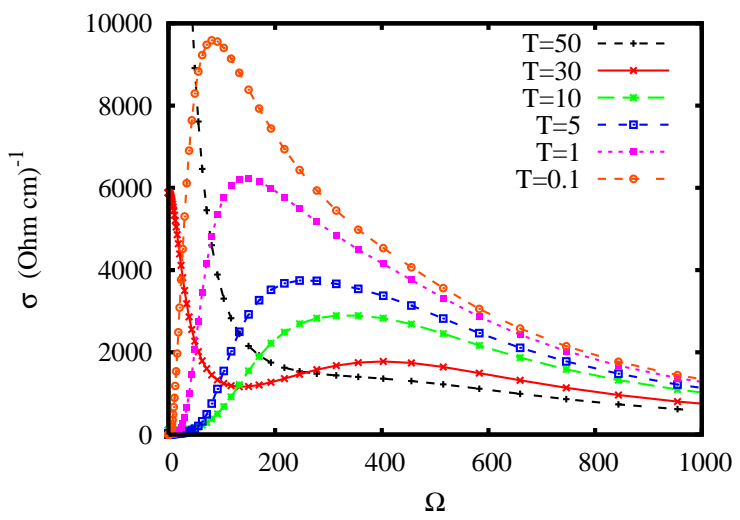

FIG. 3: (Color online) Optical conductivity for a system of QP's coupled to CM's in the quantum-critical regime $(x=$ $x_{Q C P}$ ), with $m=30 T$ and $\bar{\Omega}=30 \mathrm{~cm}^{-1}$. All frequencies and temperatures are in $\mathrm{cm}^{-1}$. The interlayer distance was taken as $d=10^{-9} \mathrm{~m}$.

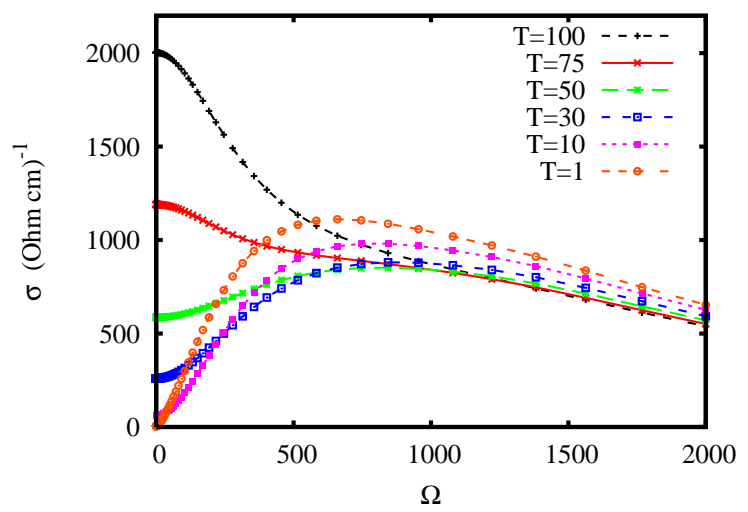

FIG. 4: (Color online) Same as Fig. 3 but with $m=3 T$ and $\bar{\Omega}=330 \mathrm{~cm}^{-1}$.

discuss a system in the quantum critical regime where the mass $m\left(T, x_{Q C P}\right) \equiv m(T)=\alpha T \underline{18}$

We first consider the case $\alpha=30$ and $\bar{\Omega}=30 \mathrm{~cm}^{-1}$, illustrated in Fig. 3. As long as $m(T) \gtrsim \bar{\Omega}$, the peak position is ruled by the critical energy scale $m$ and the strong $T$ dependence of the CM mass is visible as peaks in $\sigma(\Omega)$ at temperature-dependent frequencies: the peaks soften and sharpen upon decreasing $T$. Thermal effects tend to fill the spectra below the peaks, which are clearly visible only if the CM mass, i.e., the peak frequency, is substantially larger than $T$. We find indeed absorption peaks at frequencies $\Omega \sim m(T)$ only if the non-universal coefficient $\alpha \gtrsim 10$, as in Fig. 3 . On the other hand, in Fig. 4 we take $m(T)=3 T \lesssim \bar{\Omega} \approx 330 \mathrm{~cm}^{-1}$ and the peak position is fixed at $\bar{\Omega} \cdot \frac{1 \widetilde{9}}{\Omega}$ In this case, thermal effects show up for $T \gtrsim 0.2 \bar{\Omega}$ with absorption at low frequency. Then, the peaks are gradually embedded in this thermally-generated absorption. This shows that the absence of well-separated absorption peaks in the far infrared optical spectra does not necessarily mean that critical CM's contributing to $\sigma(\Omega)$ are absent.

- Discussion - So far we considered clean Galileaninvariant systems. The presence of a lattice introduces 
two effects: the current vertex is no longer proportional to the electron momentum and umklapp scattering processes may occur. At leading order in the critical scattering, the first effect is immaterial because the current vertex is evaluated at the HS's and the velocity only appears in multiplicative prefactors. Then, for $\bar{\Omega} \rightarrow \infty$ the Ward identities implementing the cancellation remain valid, while Eq. (5) holds for finite $\bar{\Omega}$. The second effect may instead be significant. However, our results for the leading critical contributions remain valid in those systems where $\mathbf{q}_{c}$ cannot connect Fermi surfaces in different Brillouin zones. Our analysis also provides reliable results for dirty systems in the frequency range above the typical impurity scattering rate $1 / \tau$. In assessing the results discussed above, we strongly relied on a conserving scheme to calculate $\sigma(\Omega)$. Although the relevance of vertex corrections was previously proposed ${ }^{20}$, in this Rapid Communication we provide quantitative evidence that a conserving scheme captures the strong cancellations occurring in systems with current conservation and it keeps partial cancellations also when the current conservation law is not obeyed. Therefore, non-conserving approaches in models with strong momentum dependence ${ }^{21}$ should be handled with care $\stackrel{22}{ }^{2}$

Our main result is that (the leading critical contribution to) optical absorption at finite frequency only appears, in a clean Galilean-invariant system or in systems without critical umklapp scattering, when electrons are coupled to other dynamical degrees of freedom, which mediate a retarded interaction allowing for electroncurrent dissipation. This introduces a non-universal energy scale $\bar{\Omega}$. If the CM mass $m$, controlling the distance from criticality, is larger than $\bar{\Omega}$, the CM peak position is ruled by $m$ and follows its $x$ and $T$ dependence. Otherwise, the peak is pinned at a frequency $\bar{\Omega}$. This saturation, which occurs even when $m$ vanishes with $T$
$\left(x=x_{Q C P}\right)$, is an intrinsic feature irrespective of other mechanisms (e.g., pinning), which would keep $m$ finite. Therefore, it is crucial to recognize that the saturation of the peak in far infrared spectra cannot generically be used as evidence against criticality. Thermal filling reduces the visibility of the CM peak to the regimes where the peak frequency is substantially larger than $T$. Whenever the above conditions are not fulfilled, the CM's provide broad absorption. In real systems, such absorption would interplay with the Drude peak and could be confused with an additional impurity broadening.

Experiments in cuprates provide a wealth of different behaviors, which may find counterparts in the various regimes discussed above. In $\mathrm{Bi}_{2} \mathrm{Sr}_{2} \mathrm{CuO}_{6}$ (Ref. 7) and $\mathrm{Nd}_{2-\mathrm{x}} \mathrm{Ce}_{\mathrm{x}} \mathrm{CuO}_{4-\mathrm{y}}$ (Ref. 8) peaks at temperaturedependent frequencies, clearly separated from the Drude peak, are observed, with approximate scaling behavior. Within our theory, this implies $m>\bar{\Omega}$. Peaks at temperature-independent frequencies (but still distinct from the Drude peak) are found in $\mathrm{La}_{2} \mathrm{Sr}_{\mathrm{x}} \mathrm{CuO}_{4}$ (Ref. 9), $\mathrm{La}_{1.6-\mathrm{x}} \mathrm{Nd}_{0.4} \mathrm{Sr}_{\mathrm{x}} \mathrm{CuO}_{4}$ (Ref. 10), and some $\mathrm{YBa}_{2} \mathrm{Cu}_{3} \mathrm{O}_{7-\mathrm{y}}$ (Ref. 11) samples. This behavior might be due to a non-vanishing mass or to a saturation at $\bar{\Omega}$. No peaks at all are observed in $\mathrm{Bi}_{2} \mathrm{Sr}_{2} \mathrm{Cu}_{2} \mathrm{O}_{8}$ (Ref. $\underline{6}$ ) and $\mathrm{YBa}_{2} \mathrm{Cu}_{3} \mathrm{O}_{7-\mathrm{y}}$ (Ref.12). Of course, an explicit quantitative analysis of the optical conductivity at low frequencies requires a proper treatment of disorder and umklapp processes, which is presently in progress. Nevertheless our theory accounts for the variety of experimental behaviors and may reconcile standard quantum criticality with the lack of universal scaling of the conductivity.

We acknowledge interesting discussions with C. Castellani and J. Lorenzana and financial support from the MIUR-PRIN 2005, prot. 2005022492 and from the Alexander von Humboldt foundation.
1 A. Abanov et al., Adv. Phys. 52, 119 (2003).

2 C. Castellani et al., Phys. Rev. Lett. 75, 4650 (1995); J. Phys. Chem. Solids 59, 1694 (1998).

${ }^{3}$ C. M. Varma, Phys. Rev. Lett. 83, 3538 (1999).

${ }^{4}$ W. Metzner, D. Rohe, and S. Andergassen, Phys. Rev. Lett. 91, 066402 (2003).

5 P. Coleman, Physica B 259-261, 353 (1999).

${ }^{6}$ D. van der Marel et al., Nature (London) 425, 271 (2003).

7 S. Lupi et al., Phys. Rev. B 62, 12418 (2000).

8 E. J. Singley et al., Phys. Rev. B 64, 224503 (2001).

9 A. Lucarelli et al., Phys. Rev. Lett. 90, 037002 (2003); T. Startseva et al., Physica C 321, 135 (1999).

10 M. Dumm et al., Phys. Rev. Lett. 88, 147003 (2002).

11 C. Bernhard et al., Solid State Commun. 121, 93 (2002); C. Bernhard et al., Phys. Rev. B 69, 052502 (2004).

12 J. Hwang et al., Phys. Rev. B 69, 094520 (2004); ibid. 73, 014508 (2006) and references therein.

13 P. Phillips and C. Chamon, Phys. Rev. Lett. 95, 107002 (2005).

14 G. Baym, Phys. Rev. 127, 1391 (1962).
15 G. Seibold et al., Eur. Phys. J. B 13, 87 (2000).

16 B. R. Patton and L. J. Sham, Phys. Rev. Lett. 33, 638 (1974); E. Sakai and S. Takada, Phys. Rev. B 20, 2676 (1979).

17 V. Ambegaokar, in Superconductivity, edited by P. R. Wallace (Gordon and Breach, New York, 1969).

18 S. Andergassen et al., Phys. Rev. Lett. 87, 056401 (2001); A. J. Millis, Phys. Rev. B 48, 7183 (1993).

19 This saturation always occurs in the quantum critical regime. However, in Fig. 3 owing to the low value of $\bar{\Omega}$, the saturation regime is only found at very low $T$.

20 H. Kontani et al., Phys. Rev. B 59, 14723 (1999); A. J. Millis and H. D. Drew, Phys. Rev. B 67, 214517 (2003).

21 P. Cásek et al., Phys. Rev. B 72, 134526 (2005).

22 Similar approaches in systems with momentumindependent interactions [see, e.g., F. Marsiglio et al., Phys. Lett. A 245, 172 (1998); S. V. Dordevic et al., Phys. Rev. B 71, 104529 (2005); M. R. Norman and A. V. Chubukov, ibid. 73, 140501(R) (2006)] are likely safer. 